\title{
Solving Hard ASP Programs Efficiently ${ }^{\star}$
}

\author{
Wolfgang Faber*^ and Francesco Ricca \\ Department of Mathematics, University of Calabria, \\ I-87030 Rende (CS), Italy \\ Email: \{faber,ricca\}@mat.unical.it
}

\begin{abstract}
Recent research on answer set programming (ASP) systems, has mainly focused on solving NP problems more efficiently. Yet, disjunctive logic programs allow for expressing every problem in the complexity classes $\Sigma_{2}^{P}$ and $\Pi_{2}^{P}$. These classes are widely believed to be strictly larger than NP, and several important AI problems, like conformant and conditional planning, diagnosis and more are located in this class.

In this paper we focus on improving the evaluation of $\Sigma_{2}^{P} / \Pi_{2}^{P}$-hard ASP programs. To this end, we define a new heuristic $h_{D S}$ and implement it in the (disjunctive) ASP system DLV. The definition of $h_{D S}$ is geared towards the peculiarites of hard programs, while it maintains the benign behaviour of the well-assessed heuristic of DLV for NP problems.

We have conducted extensive experiments with the new heuristic. $h_{D S}$ significantly outperforms the previous heuristic of DLV on hard 2QBF problems. We also compare the DLV system (with $h_{D S}$ ) to the QBF solvers SSolve, Quantor, Semprop, and yQuaffle, which performed best in the QBF evaluation of 2004. The results of the comparison indicate that ASP systems currently seem to be the best choice for solving $\Sigma_{2}^{P} / \Pi_{2}^{P}$ complete problems.
\end{abstract}

\section{Introduction}

Answer Set Programming (ASP) is a novel programming paradigm, which has been recently proposed in the area of nonmonotonic reasoning and logic programming. The idea of answer set programming is to represent a given computational problem by a logic program whose answer sets correspond to solutions, and then use an answer set solver to find such a solution [1]. The knowledge representation language of ASP is very expressive in a precise mathematical sense; in its general form, allowing for disjunction in rule heads and nonmonotonic negation in rule bodies, ASP can represent every problem in the complexity class $\Sigma_{2}^{P}$ and $\Pi_{2}^{P}$ (under brave and cautious reasoning, respectively) [2]. Thus, ASP is strictly more powerful than SAT-based programming, as it allows us to solve problems which cannot be translated to SAT in polynomial time. The high expressive power of ASP can be profitably exploited in AI, which often has to deal with

\footnotetext{
* This work was supported by the European Commission under projects IST-200233570 INFOMIX, and IST-2001-37004 WASP.

${ }^{\star \star}$ Funded by an APART grant of the Austrian Academy of Sciences.
} 
problems of high complexity. For instance, problems in diagnosis and planning under incomplete knowledge are complete for the complexity class $\Sigma_{2}^{P}$ or $\Pi_{2}^{P}$ $[3,4]$, and can be naturally encoded in ASP $[5,6]$.

Most of the optimization work on ASP systems has focused on the efficient evaluation of non-disjunctive programs (whose power is limited to NP/co-NP), whereas the optimization of full (disjunctive) ASP programs has been treated in fewer works (e.g., in $[7,8])$. In particular, we are not aware of any work concerning heuristics for $\Sigma_{2}^{P} / \Pi_{2}^{P}$-hard ASP programs.

Since the model generators of ASP systems, like DLV [9] and Smodels [10], are similar to the Davis-Putnam procedure, employed in many SAT solvers, the heuristic (branching rule) for the selection of the branching literal (i.e., the criterion determining the literal to be assumed true at a given stage of the computation) is fundamentally important for the efficiency of an ASP system. Some other systems, like ASSAT [11] or Cmodels [12] use a SAT solver directly as a black box, and thus have limited means of tuning the heuristic. Also note that all of Smodels, ASSAT, and Cmodels are confined to NP problems. Since our focus is on harder problems, we will not consider these systems further.

In this paper, we address the following two questions:

- Can the heuristics of ASP systems be refined to deal more efficiently with $\Sigma_{2}^{P} / \Pi_{2}^{P}$-hard ASP programs?

- On hard $\Sigma_{2}^{P} / \Pi_{2}^{P}$ problems, can ASP systems compete with other AI systems, like QBF solvers?

We define a new heuristic $h_{D S}$ for the (disjunctive) ASP system DLV. The new heuristic aims at improving the evaluation of $\Sigma_{2}^{P} / \Pi_{2}^{P}$-hard ASP programs, but it is designed to maintain the benign behaviour of the well-assessed heuristic of DLV on NP problems like 3SAT and Blocks-World, on which it proved to be very effective [13]. We experimentally compare $h_{D S}$ against the DLV heuristic on hard $2 \mathrm{QBF}$ instances, generated following recent works presented in the literature that describe transition phase results for QBFs $[14,15] . h_{D S}$ significantly outperforms the heuristic of DLV on $2 \mathrm{QBF}$.

To check the competitiveness of ASP w.r.t. QBF solvers on hard problems, we carry out an experimental comparison of the DLV system (with the new heuristic $h_{D S}$ ) with four prominent QBF solvers, which performed best at the 2004 QBF evaluation[16,17]: SSolve, Semprop, Quantor, yQuaffle. The results of the comparison, performed on instances used in the QBF competition and on a set of randomly generated instances for the Strategic Companies problem, indicate that ASP systems currently perform better than QBF systems on $\Sigma_{2}^{P} / \Pi_{2}^{P}$-hard problems.

\section{Answer Set Programming Language}

\subsection{ASP Programs}

A (disjunctive) rule $r$ is a formula

$$
a_{1} \vee \cdots \vee a_{n}:-b_{1}, \cdots, b_{k} \text {, not } b_{k+1}, \cdots, \text { not } b_{m} .
$$


where $a_{1}, \cdots, a_{n}, b_{1}, \cdots, b_{m}$ are atoms and $n \geq 0, m \geq k \geq 0$. The disjunction $a_{1} \vee \cdots \vee a_{n}$ is the head of $r$, while the conjunction $b_{1}, \cdots, b_{k}$, not $b_{k+1}, \cdots$, not $b_{m}$ is the body, $b_{1}, \cdots, b_{k}$ the positive body, and not $b_{k+1}, \cdots$, not $b_{m}$ the negative body of $r$.

An $(A S P)$ program $\mathcal{P}$ is a finite set of rules. An object (atom, rule, etc.) is called ground or propositional, if it contains no variables.

\subsection{Answer Sets}

Given a program $\mathcal{P}$, let the Herbrand Universe $U_{\mathcal{P}}$ be the set of all constants appearing in $\mathcal{P}$ and the Herbrand Base $B_{\mathcal{P}}$ be the set of all possible ground atoms which can be constructed from the predicate symbols appearing in $\mathcal{P}$ with the constants of $U_{\mathcal{P}}$.

Given a rule $r$, Ground $(r)$ denotes the set of rules obtained by applying all possible substitutions $\sigma$ from the variables in $r$ to elements of $U_{\mathcal{P}}$. Similarly, given a program $\mathcal{P}$, the ground instantiation $\operatorname{Ground}(\mathcal{P})$ of $\mathcal{P}$ is the set $\bigcup_{r \in \mathcal{P}} \operatorname{Ground}(r)$.

For every program $\mathcal{P}$, we define its answer sets using its ground instantiation $\operatorname{Ground}(\mathcal{P})$ in two steps: First we define the answer sets of positive programs, then we give a reduction of general programs to positive ones and use this reduction to define answer sets of general programs.

A set $L$ of ground literals is said to be consistent if, for every atom $\ell \in L$, its complementary literal not $\ell$ is not contained in $L$. An interpretation $I$ for $\mathcal{P}$ is a consistent set of ground literals over atoms in $B_{\mathcal{P}} \cdot{ }^{1}$ A ground literal $\ell$ is true w.r.t. $I$ if $\ell \in I ; \ell$ is false w.r.t. $I$ if its complementary literal is in $I$; $\ell$ is undefined w.r.t. $I$ if it is neither true nor false w.r.t. $I$. Interpretation $I$ is total if, for each atom $A$ in $B_{\mathcal{P}}$, either $A$ or not $A$ is in $I$ (i.e., no atom in $B_{\mathcal{P}}$ is undefined w.r.t. $I$ ). A total interpretation $M$ is a model for $\mathcal{P}$ if, for every $r \in \operatorname{Ground}(\mathcal{P})$, at least one literal in the head is true w.r.t. $M$ whenever all literals in the body are true w.r.t. $M . X$ is an answer set for a positive program $\mathcal{P}$ if it is minimal w.r.t. set inclusion among the models of $\mathcal{P}$.

Example 1. For the positive program $\mathcal{P}_{1}=\{a \vee b \vee c .,:-a\},.\{b$, not $a$, not $c\}$ and $\{c$, not $a$, not $b\}$ are the answer sets. For the positive program $\mathcal{P}_{2}=\{a \vee b \vee$ c., :-a., b:-c., c:-b. $\},\{b, c$, not $a\}$ is the only answer set.

The reduct or Gelfond-Lifschitz transform of a general ground program $\mathcal{P}$ w.r.t. an interpretation $X$ is the positive ground program $\mathcal{P}^{X}$, obtained from $\mathcal{P}$ by (i) deleting all rules $r \in \mathcal{P}$ whose negative body is false w.r.t. $\mathrm{X}$ and (ii) deleting the negative body from the remaining rules.

An answer set of a general program $\mathcal{P}$ is a model $X$ of $\mathcal{P}$ such that $X$ is an answer set of $\operatorname{Ground}(\mathcal{P})^{X}$.

\footnotetext{
${ }^{1}$ We represent interpretations as set of literals, since we have to deal with partial interpretations in the next sections.
} 


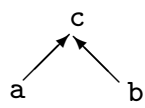

(a)

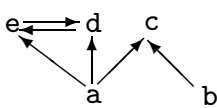

(b)

Fig. 1. Graphs (a) $D G_{\mathcal{P}_{4}}$, and (b) $D G_{\mathcal{P}_{5}}$

Example 2. Given the (general) program $\mathcal{P}_{3}=\{a \vee b:-c ., b:-\operatorname{not} a$, not c., $a \vee$ $c:-\operatorname{not} b$.$\} and I=\{b$, not $a$, not $c\}$, the reduct $\mathcal{P}_{3}^{I}$ is $\{a \vee b:-c ., b\} .$.$I is an$ answer set of $\mathcal{P}_{3}^{I}$, and for this reason it is also an answer set of $\mathcal{P}_{3}$.

\subsection{Some ASP Properties}

Given an interpretation $I$ for a ground program $\mathcal{P}$,we say that a ground atom $A$ is supported in $I$ if there exists a supporting rule $r \in \operatorname{ground}(\mathcal{P})$ such that the body of $r$ is true w.r.t. $I$ and $A$ is the only true atom in the head of $r$.

Proposition 1. [18-20] If $M$ is an answer set of a program $\mathcal{P}$, then all atoms in $M$ are supported.

Another relevant property of ASP programs is head-cycle freeness (HCF). With every ground program $\mathcal{P}$, we associate a directed graph $D G_{\mathcal{P}}=(N, E)$, called the dependency graph of $\mathcal{P}$, in which (i) each atom of $\mathcal{P}$ is a node in $N$ and (ii) there is an arc in $E$ directed from a node $a$ to a node $b$ iff there is a rule $r$ in $\mathcal{P}$ such that $b$ and $a$ appear in the head and body of $r$, respectively.

The graph $D G_{\mathcal{P}}$ singles out the dependencies of the head atoms of a rule $r$ from the positive atoms in its body. ${ }^{2}$

Example 3. Consider the program $\mathcal{P}_{4}=\{a \vee b ., c:-a ., c:-b$.$\} , and the program$ $\mathcal{P}_{5}=\mathcal{P}_{4} \cup\{d \vee e:-a ., d:-e .$, e:-d, not $b$.$\} . The dependency graph D G_{\mathcal{P}_{4}}$ of $\mathcal{P}_{4}$ is depicted in Figure 1 (a), while the dependency graph $D G_{\mathcal{P}_{5}}$ of $\mathcal{P}_{5}$ is depicted in Figure 1 (b).

The dependency graphs allow us to single out HCF programs [21]. A program $\mathcal{P}$ is $H C F$ iff there is no rule $r$ in $\mathcal{P}$ such that two atoms occurring in the head of $r$ are in the same cycle of $D G_{\mathcal{P}}$.

Example 4. The dependency graphs given in Figure 1 reveal that program $\mathcal{P}_{4}$ of Example 3 is $\mathrm{HCF}$ and that program $\mathcal{P}_{5}$ is not $H C F$, as rule $d \vee e \leftarrow$ a contains in its head two atoms belonging to the same cycle of $D G_{\mathcal{P}_{5}}$.

HCF programs are computationally easier than general (non-HCF) programs.

Proposition 2. [21, 2] 1. Deciding whether an atom belongs to some answer set of a ground HCF program $\mathcal{P}$ is NP-complete. 2. Deciding whether an atom belongs to some answer set of a ground (non-HCF) program $\mathcal{P}$ is $\Sigma_{2}^{P}$-complete.

\footnotetext{
${ }^{2}$ Note that negative literals cause no arc in $D G_{\mathcal{P}}$.
} 


\section{Answer Set Computation}

In this section, we describe the main steps of the computational process performed by ASP systems. We will refer particularly to the computational engine of the DLV system, which will be used for the experiments, but also other ASP systems, like Smodels, employ a very similar procedure.

An answer set program $\mathcal{P}$ in general contains variables. The first step of a computation of an ASP system eliminates these variables, generating a ground instantiation $\operatorname{ground}(\mathcal{P})$ of $\mathcal{P} .^{3}$ The hard part of the computation is then performed on this ground ASP program generated by the instantiator.

Function ModelGenerator(I: Interpretation): Boolean;

begin

$\mathrm{I}:=\operatorname{Det} \operatorname{Cons}(\mathrm{I})$

if $\mathrm{I}=\mathcal{L}$ then return False; $(*$ inconsistency $*$ )

if no atom is undefined in I then return IsAnswerSet(I);

Select an undefined ground atom $A$ according to a heuristic;

if ModelGenerator $(I \cup\{A\})$ then return True;

else return ModelGenerator $(I \cup\{$ not $A\})$; end;

Fig. 2. Computation of Answer Sets

The heart of the computation is performed by the Model Generator, which is sketched in Figure 2. Roughly, the Model Generator produces some "candidate" answer sets. The stability of each of them is subsequently verified by the function IsAnswerSet(I), which verifies whether the given "candidate" $I$ is a minimal model of the program $G$ round $(\mathcal{P})^{I}$ obtained by applying the GL-transformation w.r.t. $I$ and outputs the model, if so. IsAnswerSet(I) returns True if the computation should be stopped and False otherwise.

The ModelGenerator function is first called with parameter $I$ set to the empty interpretation. ${ }^{4}$ If the program $\mathcal{P}$ has an answer set, then the function returns True setting $I$ to the computed answer set; otherwise it returns False. The Model Generator is similar to the Davis-Putnam procedure employed by SAT solvers. It first calls a function DetCons(), which returns the extension of $I$ with the literals that can be deterministically inferred (or the set of all literals $\mathcal{L}$ upon inconsistency). This function is similar to a unit propagation procedure employed by SAT solvers, but exploits the peculiarities of ASP for making further inferences (e.g., it exploits the knowledge that every answer set is a minimal model). If DetCons does not detect any inconsistency, an atom $A$ is selected according to a heuristic criterion and ModelGenerator is called on $I \cup\{A\}$ and on $I \cup\{$ not $A\}$. The atom $A$ plays the role of a branching variable of a SAT solver. And indeed, like for SAT solvers, the selection of a "good" atom $A$ is crucial for the performance of an ASP system. In the next section, we describe a number of heuristic criteria for the selection of such branching atoms.

\footnotetext{
${ }^{3}$ Note that $\operatorname{ground}(\mathcal{P})$ is not the full set of all syntactically constructible instances of rules in $\mathcal{P}$; rather, it is a subset of it having precisely the same answer sets as $\mathcal{P}$.

${ }^{4}$ Observe that the interpretations built during the computation are 3-valued, that is a literal can be True, False or Undefined w.r.t. to an interpretation $I$.
} 
Remark 1. On hard ASP programs (non-hcf programs), a very large part of the computation-time may be consumed by function isAnswerSet(I), since it performs a co-NP-complete task if the program is non-hcf.

\section{Heuristics}

Throughout this section, we assume that a ground ASP program $\mathcal{P}$ and an interpretation $I$ have been fixed. Here, we describe the two heuristic criteria that will be compared in Section 5. We consider "dynamic heuristics" (the ASP equivalent of UP heuristics for $\mathrm{SAT}^{5}$ ), that is, branching rules where the heuristic value of a literal $Q$ depends on the result of taking $Q$ true and computing its consequences. Given a literal $Q, \operatorname{ext}(Q)$ will denote the interpretation resulting from the application of DetCons (see previous section) on $I \cup\{Q\}$; without loss of generality, we assume that $\operatorname{ext}(Q)$ is consistent, otherwise $Q$ is automatically set to false and the heuristic is not evaluated on $Q$ at all.

The Heuristic of DLV $\left(h_{U T}\right)$. The heuristic employed by the DLV system was proposed in [13], where it was shown to be very effective on relevant problems like 3Satisfiability, Hamilthonian Path, Blocks World, and Strategic Companies.

A peculiar property of answer sets is supportedness: For each true atom $A$ of an answer set $I$, there exists a rule $r$ of the program such that the body of $r$ is true w.r.t. $I$ and $A$ is the only true atom in the head of $r$. Since an ASP system must eventually converge to a supported interpretation, ASP systems try to keep the interpretations "as much supported as possible" during the intermediate steps of the computation. To this end, the DLV system counts the number of UnsupportedTrue (UT) atoms, i.e., atoms which are true in the current interpretation but still miss a supporting rule (further details on UTs can be found in [22] where they are called MBTs). For instance, the rule :-not $x$ implies that $x$ must be true in every answer set of the program; but it does not give a "support" for $x$. Thus, in the DLV system $x$ is taken true to satisfy the rule, and it is added to the set of UnsupportedTrue; it will be removed from this set once a supporting rule for $x$ will be found (e.g., $x \vee b$ : $-c$ is a supporting rule for $x$ in the interpretation $I=\{x$, not $b, c\})$. Given a literal $Q$, let $U T(Q)$ be the number of UT atoms in $\operatorname{ext}(Q)$. Moreover, let $U T_{2}(Q)$ and $U T_{3}(Q)$ be the number of UT atoms occurring, respectively, in the heads of exactly 2 and 3 unsatisfied rules w.r.t. $\operatorname{ext}(Q)$. The heuristic $h_{U T}$ of DLV considers $U T(Q), U T_{2}(Q)$ and $U T_{3}(Q)$ in a prioritized way, to favor atoms yielding interpretations with fewer $U T / U T_{2} / U T_{3}$ atoms (which should more likely lead to a supported model). If all UT counters are equal, then the heuristic considers the total number $\operatorname{Sat}(Q)$ of rules which are satisfied w.r.t. $\operatorname{ext}(Q)$.

The heuristic $h_{U T}$ is "balanced", that is, the heuristic values of an atom $Q$ depends on both the effect of taking $Q$ and not $Q$.

\footnotetext{
5 The UP heuristic for SAT adds for each variable $\mathrm{x}$ a unit clause $\mathrm{x}$ and $-\mathrm{x}$, respectively, and makes two independent unit propagations. The choice is then based on information thus obtained.
} 
For an atom $Q$, let $U T^{\prime}(Q)=U T(Q)+U T($ not $Q), U T_{2}^{\prime}(Q)=U T_{2}(Q)+$ $U T_{2}($ not $Q), U T_{3}^{\prime}(Q)=U T_{3}(Q)+U T_{3}(\operatorname{not} Q)$, and, eventually, $S a t^{\prime}(Q)=$ $\operatorname{Sat}(Q)+\operatorname{Sat}($ not $Q)$. Given two atoms $A$ and $B$ :

1. $A<_{h_{U T}} B$ if $U T^{\prime}(A)>U T^{\prime}(B)$;

2. otherwise, $A<_{h_{U T}} B$ if $U T^{\prime}(A)=U T^{\prime}(B)$ and $U T_{2}^{\prime}(A)>U T_{2}^{\prime}(B)$;

3. otherwise, $A<_{h_{U T}} B$ if $U T_{2}^{\prime}(A)=U T_{2}^{\prime}(B)$ and $U T_{3}^{\prime}(A)>U T_{3}^{\prime}(B)$;

4. otherwise, $A<_{h_{U T}} B$ if $U T_{3}^{\prime}(A)=U T_{3}^{\prime}(B)$ and $\operatorname{Sat}^{\prime}(A)<\operatorname{Sat}^{\prime}(B)$.

A $<{ }_{h_{U T}}$-maximum atom $A$ is selected by the heuristic $h_{U T}$ of DLV; $A$ is taken positive or negative, by comparing the values of $U T(A), U T_{2}(A), U T_{3}(A)$, and $\operatorname{Sat}(A)$, with $U T($ not $A), U T_{2}($ not $A), U T_{3}($ not $A)$, and $\operatorname{Sat}($ not $A)$, respectively, as above.

Example 5. Consider $\mathcal{P}_{6}=\{a \vee b \vee c ., d \vee e \vee f .,:-\operatorname{not} w ., w:-a ., w:-d .$, $a \vee z:-w ., b \vee z:-w .,:-d, z .,:-a, z .$,$\} , and let the current interpretation$ $I=\{w\}$; atom $w$ is UT. $a$ and $d$ are the $<_{h_{U T}}$-maxima, as only assuming their truth can eliminate the UT $w$. Indeed, anything apart from a or $d$ would be a poor choice.

The New Heuristic $\left(h_{D S}\right)$. The unsupported true atoms are, in a sense, the hardest constraints occurring in an ASP program. Indeed, as pointed out above, an unsupported true atom $x$ is intuitively like a unary constraint :-not $x$, which must be satisfied. By minimizing the UT atoms and maximizing the satisfied rules, the heuristic $h_{U T}$ tries to drive the DLV computation toward a supported model (i.e., all rules are satisfied and no UT exists). Intuitively, supported models have good chances to be answer sets (while unsupported models are guaranteed to be not answer sets), and, for simple classes of programs (e.g., tight stratified disjunctive programs) the supported models are precisely the answer sets. If the program is not tight and stratified, then supported models are not guaranteed to be answer sets; but answer-set checking can be done efficiently if the program is HCF.

For hard ASP programs (i.e., non-HCF programs - they express $\Sigma_{2}^{P}$-complete problems under brave reasoning), supported models are often not answer sets. Answer-set checking is computationally expensive (co-NP-complete), and may consume a large portion of the resources needed for computing an answer set.

The heuristic $h_{D S}$, described next, tries to drive the computation toward supported models having higher chances to be answer sets, reducing the overall number of the expensive answer-set checks. Models having a "higher degree of supportedness" are preferred, where the degree of supportedness is the average number of supporting rules for the true atoms (note that this number is higher than one, on supported models). Intuitively, if all true atoms have many supporting rules in a model $M$, then the elimination of an atom from the model would violate many rules, and it becomes less likely finding a subset of $M$ which is a model of $\mathcal{P}^{M}$, to disprove that $M$ is an answer set.

We next formalize this intuition to define the new heuristic $h_{D S}$. Given a literal $Q$, let $\operatorname{True}(Q)$ be the number of true non-HCF atoms in $\operatorname{ext}(Q)$, and let 
SuppRules $(Q)$ be the number of all supporting rules for non-HCF atoms w.r.t. $\operatorname{ext}(Q)$. Intuitively, the heuristic maximizes the "degree of supportedness" of the interpretation, intended as the ratio between the number of supporting rules and the number of true atoms. Also in this case, the heuristic is "balanced", it takes into account both the atom and its complement.

Moreover, it is defined as a refinement of the heuristic $h_{U T}$ (i.e., $A<_{h_{U T}} B \Rightarrow$ $\left.A<_{h_{D S}} B\right)$. In this way, $h_{D S}$ keeps the same nice behaviour as the well-assessed $h_{U T}$ on NP problems like $3 \mathrm{SAT}$ and Blocks-World, where $h_{U T}$ proved to be very effective [13]; while, as we will see in Section 5 it sensibly improves on $h_{U T}$ on hard 2QBF problems ( $\Sigma_{2}^{P}$-complete). Given two atoms $A$ and $B$ :

1. $A<_{h_{D S}} B$ if $A<_{h_{U T}} B$;

2. otherwise, $A<_{h_{D S}} B$ if $B \nless_{h_{U T}} A$ and $D S(A)<D S(B)$

where $D S(Q)=\operatorname{SuppRules}(Q) / \operatorname{True}(Q)+\operatorname{SuppRules}(\operatorname{not} Q) / \operatorname{True}(\operatorname{not} Q){ }^{6}$

The heuristic selects a $<_{h_{D S}}$-maximum atom $A ; A$ is taken positive or negative, by comparing the degree of supportedness of $A$ and not $A$.

Example 6. Reconsider Example 5 with the interpretation being $I=\{w\}$. We get $\operatorname{ext}(a)=\{w, a, b, \operatorname{not} z, \operatorname{not} c\}, \operatorname{ext}(d)=\{w, d, a, b, \operatorname{not} z$, not $c$, not $e$, not $f\}$. $D S(a)=3 / 3$, since $w \leftarrow a ; a \vee z \leftarrow w$ and $b \vee z \leftarrow w$ are supporting rules for the three true non-HCF atoms $w, a, b$. On the other hand, $D S(d)=4 / 3$, since $w \leftarrow d$ is an additional supporting rule for the same three true non-HCF atoms $w, a, b$. Therefore $a<_{h_{D S}} d$ holds. Indeed, $d$ is a better choice than a, as it leads immediately to an answer set. a would require at least another choice, and choosing e or $f$ would cause a failing model check.

\section{Comparing $h_{U T}$ vs $h_{D S}$ : Experiments}

The proposed heuristic aims at improving the performance of DLV on hard $\left(\Sigma_{2}^{P}-\right.$ complete) ASP programs. While there are many experimental works benchmarking ASP systems on NP-complete problems, less is available for $\Sigma_{2}^{P}$-complete problems. We resort to $2 \mathrm{QBF}$, the canonical problem, and one of the few $\Sigma_{2}^{P}$ hard problems for which some transition phase results are known $[14,15]$.

The problem here is to decide whether a quantified Boolean formula (QBF) $\Phi=\exists X \forall Y \phi$, where $X$ and $Y$ are disjoint sets of propositional variables and $\phi=C_{1} \vee \ldots \vee C_{k}$ is a $3 \mathrm{DNF}$ formula over $X \cup Y$, is valid. The transformation from $2 \mathrm{QBF}$ to disjunctive logic programming is based on a reduction used in [23]. The propositional disjunctive logic program $\mathcal{P}_{\phi}$ produced by the transformation requires $2 *(|X|+|Y|)+1$ predicates (with one dedicated predicate $w$ ).

Our benchmark instances were generated following recent works presented in the literature that describe transition phase results for QBFs [14,15], see [9], for a thorough discussion. In all generated instances, the number of $\forall$-variables in any formula is the same as the number of $\exists$-variables (that is, $|X|=|Y|$ ) and each disjunct contains at least two universal variables. Moreover, the number of clauses is $((|X|+|Y|) / 2)^{0.5}$.

\footnotetext{
${ }^{6}$ The denominator is increased by 1 , in order to avoid possible divisions by zero.
} 

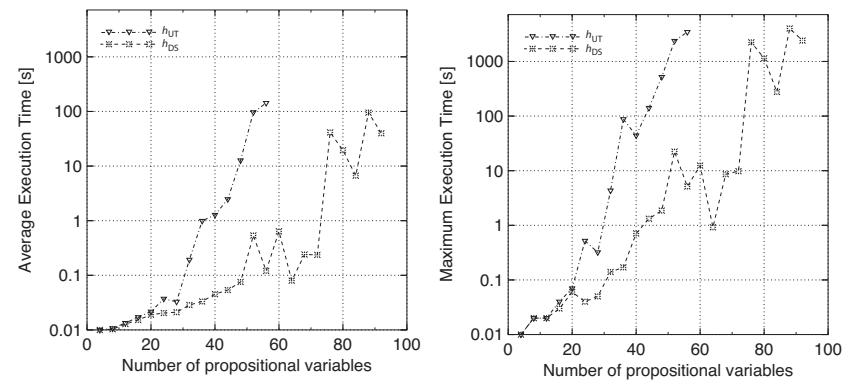

Fig. 3. Running Times on Random QBF problems

Experiments were performed on a PentiumIV $1500 \mathrm{MHz}$ machine with $256 \mathrm{MB}$ RAM running SuSe Linux 9.0. Time measurements have been done using the time command shipped with SuSe Linux 9.0.

We generated 100 random QBF instances for each problem size. The results of our experiments are displayed in Fig. 3. For each instance, we allowed a maximum time of 7200 seconds (two hours). The line of a system stops whenever some problem instance was not solved within this time limit. On the vertical axis, we report, respectively, the average and the maximum running time in seconds over the 100 instances of the respective size, in logarithmic scale.

It is evident that the new heuristic $h_{D S}$ outperforms the heuristic $h_{U T}$ in these experiments. Heuristic $h_{U T}$ stopped at size 56 ; while heuristic $h_{D S}$ solved all instances up to size 92 . To solve an instance of size $56, h_{U T}$ took $3455.85 \mathrm{~s}$; while $h_{D S}$ required at most $5.13 \mathrm{~s}$ and $0.12 \mathrm{~s}$ on average for instances of this size. Heuristic $h_{U T}$ could not solve a 60-variables instance within 2 hours of cpu time; while $h_{D S}$ took at most $12.41 \mathrm{~s}$ and $0.64 \mathrm{~s}$ on average for solving these.

\section{ASP vs QBF Solvers}

One may wonder whether ASP systems are competitive with other systems on $\Sigma_{2}^{P} / \Pi_{2}^{P}$-hard problems. Currently it seems that QBF solvers are the most prominent (and efficient) non-ASP-systems for such problems.

In order to answer this question, we carry out an experimental comparison of DLV (with the heuristic described in this paper) with QBF solvers which performed best at the $2004 \mathrm{QBF}$ evaluation [16,17]: SSolve [24] (in the version used at the 2004 QBF evaluation), Semprop [25] (version v01.06.04), Quantor [26] (version 1.3pre1), and yQuaffle [27] (version 093004). We use two different sets of benchmarks, which we describe in the following sections.

\subsection{QBF Evaluation}

The first group of benchmarks constitute the $\Sigma_{2}^{P}$ - and $\Pi_{2}^{P}$-complete QBF instances of the 2004 QBF evaluation, which we obtained from the qbflib web site [16]. These instances are of four different kinds: (1) Letz-tree, (2) Narizzanorobot, (3) Pan-Kph, and (4) hard random-instances, see [16] for details. In total, our suite contains 143 2QBF instances: 2 Letz-tree, 32 Narizzano-robot, 1 Pan-Kph, and 108 random instances. For DLV we used a standard propositional 


\begin{tabular}{|c|c|c|c|c|c|}
\hline & $\mathrm{DLV}$ & Quantor & Semprop & yQuaffle & SSolve \\
\hline Robot & $32(100 \%)$ & $10(31 \%)$ & $17(53 \%)$ & $21 \quad(67 \%)$ & $22 \quad(69 \%)$ \\
\hline Random & $108(100 \%)$ & $14(13 \%)$ & $96 \quad(89 \%)$ & $55 \quad(51 \%)$ & $103 \quad(95 \%)$ \\
\hline Tree & $2(100 \%)$ & $2(100 \%)$ & $2(100 \%)$ & $2(100 \%)$ & $2(100 \%)$ \\
\hline$P a n-K p h$ & $1(100 \%)$ & $1(100 \%)$ & $1(100 \%)$ & $1(100 \%)$ & $1(100 \%)$ \\
\hline Total & $143(100 \%)$ & $27(19 \%)$ & $116(81 \%)$ & 79 (55\%) & $(90 \%)$ \\
\hline
\end{tabular}

Table 1. Number (and percentage) of instances solved within the allowed time.

encoding as described in Sec. 5, while for the QBF systems we used directly the qDimacs format.

The experiments were performed on the same machine as those of Sec. 5. For each instance, we have allowed a maximum running time of 1800 seconds (30 minutes). Again, we have limited the process size to $256 \mathrm{MB}$ to avoid swapping.

Table 1 displays, for each system, the number and percentage of instances which have been solved under the resource limitations. Summarizing, DLV could solve all instances (100\%) and is therefore clearly the best among the compared systems. Among the QBF solvers, SSolve and Semprop could solve $81 \%$ and $88 \%$ of the instances, respectively, and thus performed significantly better than both yQuaffle (55\%) and Quantor (19\%). It should be noted that practically all of the unsolved instances for Quantor are due to excessive memory consumption, while for the other systems they are due to time-outs. Indeed, we have tried to run Quantor on some of its unsolved instances manually: Within the first minute of CPU time (several minutes real-time due to swapping), it had typically allocated around $500 \mathrm{MB}$, and after two minutes (around half an hour in real time) more than $700 \mathrm{MB}$, still growing. We then aborted the test to avoid a machine lock-up.

\begin{tabular}{|c|r|r|r|r|}
\hline & SSolve & Semprop & Quantor & yQuaffle \\
\hline \# solved & 128 & 116 & 27 & 79 \\
\hline solver avg & 43,86 & 68,18 & 4,74 & 55,24 \\
DLV avg & 38,95 & 43,50 & 10,94 & 49,05 \\
\hline
\end{tabular}

Table 2. Average time (seconds) on instances solved by QBF systems

While SSolve and Semprop did significantly better on the random instances than on the "Narizzano-robot" instances, the situation is inverse for Quantor and yQuaffle, which confirms the observations in [17].

Also when comparing the average runtime between DLV and each QBF solver (on the instances solved by the respective system), DLV usually has an edge, as Table 2 shows. The average runtime of DLV is only larger when comparing to Quantor; but given that this comparison is based only on $19 \%$ of all instances, this is rather insignificant.

\subsection{Strategic Companies}

The second group of benchmarks is made up of randomly generated instances for the Strategic Companies problem, as defined in [28]. We use the same DLV program and generation method as in [9].

Here, we generated tests as in [9] with 20 instances each size for $m$ companies $(5 \leq m \leq 200), 3 m$ products, 10 uniform randomly chosen contr_by relations 
per company (up to four controlling companies), and uniform randomly chosen prod_by relations (up to four producers per product). The problem is deciding whether two fixed companies (1 and 2, without loss of generality) are strategic.

For the QBF solvers we have produced the following formula: $\exists c_{1}, \ldots, c_{n}$ : $\forall c_{1}^{\prime}, \ldots, c_{n}^{\prime}:\left((I \wedge N E) \rightarrow\left(R \wedge R^{\prime}\right) \wedge c_{1} \wedge c_{2}\right)$ where $I$ stands for $\left(c_{1}^{\prime} \rightarrow c_{1}\right) \wedge \ldots \wedge$ $\left(c_{n}^{\prime} \rightarrow c_{n}\right), N E$ for $\neg\left(\left(c_{1}^{\prime} \leftrightarrow c_{1}\right) \wedge \ldots \wedge\left(c_{n}^{\prime} \leftrightarrow c_{n}\right)\right), R$ for $\bigwedge_{i=1}^{m}\left(\left(\bigwedge_{c_{j} \in O_{i}} c_{j}\right) \rightarrow\right.$ $\left.c_{i}\right) \wedge \bigwedge_{i=1}^{n}\left(\bigvee_{g_{i} \in C_{j}} c_{j}\right)\left(O_{i}\right.$ contains the controlling companies of $c_{i}$, while $C_{j}$ contains the companies producing good $j . R^{\prime}$ is defined analogous to $R$ on the primed variables.

Unfortunately this formula is not in $\mathrm{CNF}$, as required by the qDimacs format. In order to avoid a substantial blowup of the formula by a trivial normalization, we have used the tool qst of the traquasto suite [29], which transforms a formula into qDimacs by introducing additional "label variables" to avoid exponential formula growth. However, these additional variables are existentially quantified at the inner level and thus would turn the formula above into a $3 \mathrm{QBF}$. To avoid this, we consider the negated formula $\forall c_{1}, \ldots, c_{n}: \exists c_{1}^{\prime}, \ldots, c_{n}^{\prime}: \neg((I \wedge N E) \rightarrow$ $\left.\left(R \wedge R^{\prime}\right) \wedge c_{1} \wedge c_{2}\right)$, which stays on the second level after the transformation.
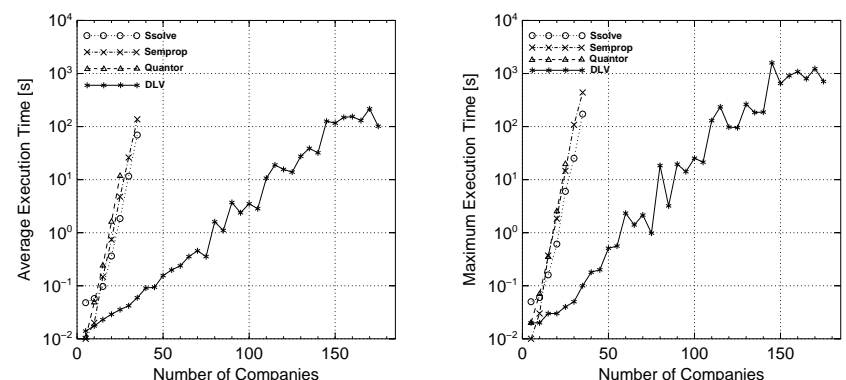

Fig. 4. Average (left) and maximum (right) timings for Strategic Companies

In the same experimental setting as before, we obtained the results of Fig. $4 .{ }^{7}$ It is evident that DLV scales significantly better than the QBF solvers (note that the vertical axis is logarithmic), and can solve all instances of up to 175 companies, while the QBF solver fail to solve instances of 40 companies.

\section{Conclusion}

In this paper, we have presented a new heuristic method for ASP systems, which is geared towards hard problems on the second level of the polynomial hierarchy. We have implemented this method in the state-of-the-art system DLV, and showed that it is beneficial for the performance of the system.

To our knowledge, this is the first work dealing with heuristics dedicated for $\Sigma_{2}^{P} / \Pi_{2}^{P}$-hard ASP programs. Previous optimization techniques for this segment have been concerned with the model checking portion, which is important for this class of problems. In our work, we attack the problem earlier, in the model

\footnotetext{
${ }^{7}$ yQuaffle is not included, as it triggered assertions on some of the input files.
} 
generation phase, and can therefore cut on the model checks. Importantly, this heuristics has been incorporated in a way such that the benign behavior on $\mathrm{NP}$ /co-NP programs w.r.t. the previous heuristic of DLV is maintained.

We experimentally verified that the new heuristic significantly improves the DLV system performance on randomly generated hard 2QBF instances, reducing the average execution time, enlarging the maximum solvable size of these problems for a fixed time limit.

We also carried out an experimental comparison of DLV (with the heuristic described in this paper) with the best QBF solvers of the 2004 QBF evaluation [16, 17]: SSolve [24], Semprop [25], Quantor [26], and yQuaffle [27]. This comparison was done on benchmark instances of the 2004 QBF evaluation, and Strategic Companies. In both cases, DLV could outperform the QBF solvers, often significantly. DLV was able to solve all the instances of the 2004 QBF evaluation within the given resource limitations, while the best QBF system solved $88 \%$, and the worst only 19\%. Also for Strategic Companies, DLV exhibited much better performance. We therefore conclude that ASP systems are currently the best choice for solving $\Sigma_{2}^{P} / \Pi_{2}^{P}$-complete problems. All benchmark data is available at http://www.dlvsystem. com/examples/tests-sigma2-2005.tar.gz.

We note again that QBF solvers are designed for solving also harder problems than the ones considered here. Nevertheless, they are used for solving problems of this kind, especially planning problems, cf. [30]. However, from our results we have to conclude that DLV appears to be the better choice for $\Sigma_{2}^{P} / \Pi_{2}^{P}$-complete problems

\section{References}

1. Lifschitz, V.: Answer Set Planning. In Schreye, D.D., ed.: ICLP'99, Las Cruces, New Mexico, USA, The MIT Press (1999) 23-37

2. Eiter, T., Gottlob, G., Mannila, H.: Disjunctive Datalog. ACM TODS 22 (1997) 364-418

3. Rintanen, J.: Improvements to the Evaluation of Quantified Boolean Formulae. In Dean, T., ed.: IJCAI 1999, Sweden,(1999) 1192-1197

4. Eiter, T., Gottlob, G.: The Complexity of Logic-Based Abduction. JACM 42 (1995) 3-42

5. Baral, C.: Knowledge Representation, Reasoning and Declarative Problem Solving. CUP (2002)

6. Leone, N., Rosati, R., Scarcello, F.: Enhancing Answer Set Planning. In: IJCAI01 Workshop on Planning under Uncertainty and Incomplete Information. (2001) $33-42$

7. Janhunen, T., Niemelä, I., Simons, P., You, J.H.: Partiality and Disjunctions in Stable Model Semantics. In: KR 2000, 12-15,(2000) 411-419

8. Koch, C., Leone, N., Pfeifer, G.: Enhancing Disjunctive Logic Programming Systems by SAT Checkers. Artificial Intelligence 15 (2003) 177-212

9. Leone, N., Pfeifer, G., Faber, W., Eiter, T., Gottlob, G., Perri, S., Scarcello, F.: The DLV System for Knowledge Representation and Reasoning. ACM TOCL (2005) To appear. 
10. Simons, P., Niemelä, I., Soininen, T.: Extending and Implementing the Stable Model Semantics. Artificial Intelligence 138 (2002) 181-234

11. Lin, F., Zhao, Y.: ASSAT: Computing Answer Sets of a Logic Program by SAT Solvers. In: AAAI-2002, Edmonton, Alberta, Canada, AAAI Press / MIT Press (2002)

12. Lierler, Y., Maratea, M.: Cmodels-2: SAT-based Answer Set Solver Enhanced to Non-tight Programs. In: LPNMR-7. LNCS, (2004) 346-350

13. Faber, W., Leone, N., Pfeifer, G.: Experimenting with Heuristics for Answer Set Programming. In: IJCAI 2001, Seattle, WA, USA,(2001) 635-640

14. Cadoli, M., Giovanardi, A., Schaerf, M.: Experimental Analysis of the Computational Cost of Evaluating Quantified Boolean Formulae. In: AI*IA 97. Italy, (1997) $207-218$

15. Gent, I., Walsh, T.: The QSAT Phase Transition. In: AAAI. (1999)

16. Narizzano, M., Tacchella, A.: QBF Solvers Evaluation page (2002) http://www. qbflib.org/qbfeval/index.html/.

17. Berre, D.L., Simon, L., Tacchella, A.: Challenges in the QBF Arena: the SAT'03 Evaluation of QBF Solvers. In: SAT'03. Santa Margherita Ligure, Italy, (2003) 468-485

18. Marek, W., Subrahmanian, V.: The Relationship between Logic Program Semantics and Non-Monotonic Reasoning. In: ICLP'89, MIT Press (1989) 600-617

19. Leone, N., Rullo, P., Scarcello, F.: Disjunctive Stable Models: Unfounded Sets, Fixpoint Semantics and Computation. Information and Computation 135 (1997) 69-112

20. Baral, C., Gelfond, M.: Logic Programming and Knowledge Representation. JLP 19/20 (1994) 73-148

21. Ben-Eliyahu, R., Dechter, R.: Propositional Semantics for Disjunctive Logic Programs. AMAI 12 (1994) 53-87

22. Faber, W., Leone, N., Pfeifer, G.: Pushing Goal Derivation in DLP Computations. In: LPNMR'99. LNCS 1730

23. Eiter, T., Gottlob, G.: On the Computational Cost of Disjunctive Logic Programming: Propositional Case. AMAI 15 (1995) 289-323

24. Feldmann, R., Monien, B., Schamberger, S.: A Distributed Algorithm to Evaluate Quantified Boolean Formulae. In: Proceedings National Conference on AI (AAAI'00), Austin, Texas, AAAI Press (2000) 285-290

25. Letz, R.: Lemma and Model Caching in Decision Procedures for Quantified Boolean Formulas. In: TABLEAUX 2002. Denmark, (2002) 160-175

26. Biere, A.: Resolve and Expand. (2004) SAT'04.

27. Zhang, L., Malik, S.: Towards a Symmetric Treatment of Satisfaction and Conflicts in Quantified Boolean Formula Evaluation. In: CP 2002. NY, USA, (2002) 200-215

28. Cadoli, M., Eiter, T., Gottlob, G.: Default Logic as a Query Language. IEEE TKDE 9 (1997) 448-463

29. Zolda, M.: Comparing Different Prenexing Strategies for Quantified Boolean Formulas. Master's thesis, TU Wien (2005)

30. Castellini, C., Giunchiglia, E., Tacchella, A.: SAT-based planning in complex domains: Concurrency, constraints and nondeterminism. Artificial Intelligence 147 (2003) 85-117 